TRANSACTIONS OF THE

AMERICAN MATHEMATICAL SOCIETY

Volume 349, Number 5, May 1997, Pages 1945-1959

S 0002-9947(97)01797-2

\title{
PRINCIPAL EIGENVALUES WITH INDEFINITE WEIGHT FUNCTIONS
}

\author{
ZHIREN JIN
}

\begin{abstract}
Both existence and non-existence results for principal eigenvalues of an elliptic operator with indefinite weight function have been proved. The existence of a continuous family of principal eigenvalues is demonstrated.
\end{abstract}

\section{INTRODUCTION AND RESULTS}

Let $g(x)$ be a locally Hölder continuous function on $R^{n}$ which changes sign. We consider the following eigenvalue problem with indefinite weight:

$$
-\Delta u(x)=\lambda g(x) u(x) \text { for } x \in R^{n},
$$

where $\Delta$ is the standard Laplace operator. A principal eigenvalue of $(P)$ is a positive constant $\lambda_{0}$, such that we can find a positive solution $u(x)$ for $(P)$ if $\lambda=\lambda_{0}$.

Recently, a number of authors have investigated the existence of principal eigenvalues for $(P)$. Part of the motivation to study $(P)$ is to understand the bifurcation behavior of solutions for some nonlinear problems arising in population genetics [4], [5], [7]. Brown, Cosner and Fleckinger in [3] showed that if $\int_{R^{n}} g(x) d x>0$, $n=1,2$, then $(P)$ has no principal eigenvalues, while if $n \geq 3$ and $g(x)$ is negative and bounded away from 0 near $\infty$, then $(P)$ has a principal eigenvalue. Brown and Tertikas [6] improved the results in [3] to conclude that $(P)$ has a principal eigenvalue if $g^{+}(x)=\max \{g(x), 0\}$ has compact support. When $g(x)$ is bounded and $g^{+}(x) \in L^{\frac{n}{2}}\left(R^{n}\right)$, the existence of one principal eigenvalue and infinitely many other eigenvalues was proved by Allegretto in [1].

The goal of this paper is to continue to investigate when $(P)$ has a principal eigenvalue. We shall show the existence of not only one principal eigenvalue, but also a continuous family of principal eigenvalues. Indeed by applying our results to the case that $g^{+}(x)=\max \{g(x), 0\}$ has compact support, we can conclude that there is a number $\lambda_{0}>0$, such that any number $\lambda$ is a principal eigenvalue of $(P)$ as long as $0<\lambda \leq \lambda_{0}$.

The basic idea in the paper is to compare the different cases with different weight functions $g(x)$. First let us introduce a definition:

$(G)$ : A non-negative function $g_{1}(x)$ on $R^{n}$ is said to have the property $(G)$ if it is not identically zero and there is a principal eigenvalue $\mu$ for the problem

$$
-\Delta u(x)=\mu g_{1}(x) u(x) \quad \text { for } x \in R^{n} .
$$

Received by the editors April 3, 1995 and, in revised form, November 20, 1995.

1991 Mathematics Subject Classification. Primary 35J65, 35J25.

Key words and phrases. Principal eigenvalue, Laplace operator, indefinite weight function, conformal deformation on the unit sphere, maximum principles.

(C)1997 American Mathematical Society 
Our first result says that $(P)$ has a continuous family of principal eigenvalues if the weight function is dominated by a function having the property $(G)$.

Theorem 1. Let $n \geq 3$, and let $g_{1}(x)$ be a non-negative function having the prop$\operatorname{erty}(G)$. If

$$
g(x) \leq g_{1}(x) \quad \text { for } \quad x \in R^{n},
$$

then any number $\lambda$ is a principal eigenvalue of $(P)$ as long as $0<\lambda \leq \mu$, where $\mu$ is the number defined in the property $(G)$.

By Theorem 1, if we can find a function $g_{1}(x)$ having the property $(G)$, we can prove the existence of principal eigenvalues for all weight functions dominated by $g_{1}(x)$. If $n=2$, the results in [3] showed that there are no functions having the property $(G)$. When $n \geq 3$, there are a lot of functions having the property $(G)$. Indeed the following result is part of Theorem 1 in [1] (since no information on the asymptotic behavior of eigenfunctions is given in the following result, one does not need to assume that $g(x)$ is globally bounded as in Theorem 1 in [1]).

Theorem 2 (Theorem 1 in [1]). If $n \geq 3, g_{1}(x) \geq 0$ is not identically zero and

$$
\int_{R^{n}}\left|g_{1}(x)\right|^{\frac{n}{2}} d x<\infty,
$$

then $g_{1}(x)$ has the property $(G)$ and

$$
\mu \geq c(n)\left(\int_{R^{n}}\left|g_{1}(x)\right|^{\frac{n}{2}} d x\right)^{-\frac{2}{n}},
$$

where $c(n)$ is a constant depending only on the dimension $n$ and $\mu$ is the principal eigenvalue defined in the property $(G)$.

As an application of Theorems 1 and 2, we have (we recall $g^{+}(x)=\max \{g(x), 0\}$ )

Theorem 3. If $n \geq 3, g\left(x_{0}\right)>0$ for some $x_{0} \in R^{n}$, and

$$
\int_{R^{n}}\left|g^{+}(x)\right|^{\frac{n}{2}} d x<\infty
$$

then any number $\lambda$ is a principal eigenvalue of $(P)$ as long as

$$
0<\lambda \leq c(n)\left(\int_{R^{n}}\left|g^{+}\right|^{\frac{n}{2}} d x\right)^{-\frac{2}{n}},
$$

where $c(n)$ is a constant depending only on the dimension $n$.

Proof. By the assumption and Theorem 2, $g^{+}(x)$ has the property $(G)$ and $g(x)$ is naturally dominated by $g^{+}(x)$. Then Theorem 1 concludes the proof.

Comparing Theorem 3 with results in [1], [2], [5], we have generalized the existence results in [1], [2], [5] to the existence of a continuous family of principal eigenvalues. Furthermore, if $\int_{R^{n}} g(x) d x>0, n \geq 3,(P)$ still has principal eigenvalues provided the conditions of Theorem 3 are satisfied. This is in contrast to the results in [3], which showed non-existence of principal eigenvalues if $n=1,2$ and $\int_{R^{n}} g(x) d x>0$.

To apply Theorem 1, it is important to find out which functions have the property $(G)$. Here is a trivial way to produce more functions having the property $(G)$. Let $g_{1}\left(x_{1}, x_{2}, x_{3}\right)$ be a function defined on $R^{3}$ having the property $(G)$. Then there is a 
positive constant $\mu$ and a positive function $u(x)$, such that $-\Delta u(x)=\mu g_{1}(x) u(x)$ for $x \in R^{3}$. Now we extend $u(x)$ and $g_{1}(x), x \in R^{3}$, onto $R^{n}$ trivially, that is,

$$
u\left(x_{1}, x_{2}, x_{3}, \cdots, x_{n}\right)=u\left(x_{1}, x_{2}, x_{3}\right)
$$

and

$$
g_{1}\left(x_{1}, x_{2}, x_{3}, \cdots, x_{n}\right)=g_{1}\left(x_{1}, x_{2}, x_{3}\right) .
$$

Then we see that $-\Delta u(x)=\mu g_{1}(x) u(x)$ for $x \in R^{n}$. Thus $g_{1}(x)$ is a function on $R^{n}$ having the property $(G)$. Therefore, from Theorem 1, we have

Theorem 4. Assume that $n \geq 3$, and there is a non-negative function $g_{1}\left(x_{1}, x_{2}, x_{3}\right)$ having the property $(G)$, such that

$$
g\left(x_{1}, x_{2}, x_{3}, \cdots, x_{n}\right) \leq g_{1}\left(x_{1}, x_{2}, x_{3}\right) \quad \text { on } \quad R^{n} .
$$

Then any number $\lambda$ is a principal eigenvalue of $(P)$ as long as $0<\lambda \leq \mu$, where $\mu$ is defined in the property $(G)$.

An immediate consequence of Theorem 4 is that there are functions having the property $(G)$ and satisfying $\int_{R^{n}}\left|g^{+}\right|^{\frac{n}{2}} d x=\infty(n \geq 4)$. Therefore there are functions having the property $(G)$ and not covered by Theorem 2 . The next result gives more functions having the property $(G)$.

Theorem 5. Let $n \geq 3$, and let $g_{1}(x)$ be a non-negative, not identically zero function on $R^{n}$ and

$$
g_{1}(x) \leq \frac{c}{|x|^{2}} \quad \text { for } \quad x \in R^{n} .
$$

Then $g_{1}(x)$ has the property $(G)$, and we can take $\mu=\frac{(n-2)^{2}}{4 c}$.

Remark 1.1. From Theorem 5, we see that

$$
g_{1}\left(x^{\prime}\right)=\left(1+\left|x^{\prime}\right|^{2}\right)^{-1} \quad \text { with } x^{\prime}=\left(x_{1}, x_{2}, x_{3}\right)
$$

has the property $(G)$. Then by applying Theorems 1,4 and 5 , we conclude that for all $g(x)$, if $g(x) \leq g_{1}\left(x^{\prime}\right)$ on $R^{n}$, then $(P)$ has a continuous family of principal eigenvalues.

The next result says that the condition in Theorem 5 is quite sharp.

Theorem 6. If $n \geq 3$, and

$$
\lim _{|x| \longrightarrow \infty} g(x)|x|^{2}=\infty,
$$

then $(P)$ has no principal eigenvalues.

Remark 1.2. Theorem 6 is also true if $n=2$. Indeed, in this case we automatically get $\int_{R^{2}} g(x) d x=\infty$; then we can use the result in [3].

In Theorems 1-5, we have no specific control on the asymptotic behavior of eigenfunctions. To obtain control on the asymptotic behavior of eigenfunctions, we need to add additional assumptions on the weight function.

Theorem 7. Assume that $n \geq 3, \int_{R^{n}}\left|g^{+}\right|^{\frac{n}{2}} d x<\infty, g\left(x_{0}\right)>0$ for some $x_{0} \in R^{n}$, and there is a $p>\frac{n}{2}$ such that

$$
\int_{R^{n}}|g|^{p}\left(1+|x|^{2}\right)^{2 p-n} d x<\infty
$$


Then $(P)$ has a principal eigenvalue $\lambda_{0}$ and an eigenfunction $u(x)$ such that

$$
\lambda_{0} \geq c(n)\left(\int_{R^{n}}\left|g_{1}^{+}\right|^{\frac{n}{2}} d x\right)^{-\frac{2}{n}}
$$

where $c(n)$ is a constant depending only on the dimension n. Moreover,

$$
u(x)|x|^{n-2} \longrightarrow c_{0} \quad \text { as } \quad|x| \longrightarrow \infty \text { for a non-negative constant } c_{0} .
$$

Remark 1.3. If there are $K>0$ and $\alpha>2$ such that $|g(x)| \leq K|x|^{-\alpha}$ for $|x|$ large, the existence of a principal eigenvalue with an eigenfunction $u(x)(u(x) \longrightarrow 0$ as $|x| \longrightarrow \infty)$ was shown in Theorem 4.1 in [3]. In this case, the assumptions of Theorem 7 are clearly satisfied. If $g(x)$ is bounded and $g^{+}(x) \in L^{\frac{n}{2}}\left(R^{n}\right)$, the existence of a principal eigenvalue with an eigenfunction $u(x)(u(x) \longrightarrow 0$ as $|x| \longrightarrow \infty)$ was shown in [1]. In Theorem 7, we do not assume $g(x)$ is bounded. Another comment is that Theorem 7 only yields the asymptotic behavior of one eigenfunction corresponding to a particular principal eigenvalue. By Theorem 3, under the assumptions of Theorem 7, $(P)$ has a continuous family of principal eigenvalues. Most likely the asymptotic behavior of other eigenfunctions is not the same as the one described in (1.1) (see Remark 2.2 for some examples).

Finally, we consider the case $n=2$. By a result in [3], a necessary condition for $(P)$ having a principal eigenvalue is $\int_{R^{2}} g(x)<0$. We would like to find out if this condition is sufficient. Although we believe the answer is yes, we only can prove a weaker result here.

Theorem 8. Assume that $n=2, \int_{R^{2}} g(x) d x<0, g\left(x_{0}\right)>0$ for some $x_{0} \in R^{2}$, and there is a $p>2$ such that

$$
\int_{R^{2}}\left|g^{+}\right|^{p}\left(1+|x|^{2}\right)^{2(p-1)} d x<\infty .
$$

Then $(P)$ has a principal eigenvalue $\lambda_{0}$.

Remark 1.4. It would be interesting to know if there is also a continuous family of principal eigenvalues for the case $n=2$. We can not do this here, since by a result in [3] there is no function having the property $(G)$ for $n=2$.

The paper is organized as follows. In section 2 , we consider the case $n \geq 3$, where Theorems $1,2,5,6$, and 7 are proved and a generalization to more general elliptic equations is given. In section 3 , we consider the case $n=2$, where Theorem 8 is proved.

\section{ACKNOWLEDGEMENT}

The work is partially supported by a LAS Summer Research Fellowship at Wichita State University.

\section{THE CASE THAT $n \geq 3$}

We first prove Theorem 1.

Proof of Theorem 1. By assumption, there is a positive number $\mu$ and positive function $w(x)$ on $R^{n}$, such that

$$
-\Delta w=\mu g_{1}(x) w \quad \text { on } \quad R^{n} .
$$


Let $B_{k}(0)$ be the ball centered at origin with radius $k, k=1,2,3, \cdots$. For each fixed number $0<\lambda \leq \mu$, we consider a family of boundary value problems:

$$
\left\{\begin{array}{lll}
-\Delta u=\lambda g(x) u \quad \text { on } & B_{k}(0) \\
u=k & \text { on } \partial B_{k}(0)
\end{array}\right.
$$

We claim that $\left(P_{k}\right)$ has a positive solution $u_{k}(x)$ for all $k$.

We set $u_{k}(x)=v_{k}(x) w(x)$; then it is straightforward to check (using (2.1) and $\left.\left(P_{k}\right)\right)$ that $u_{k}(x)$ is a positive solution of $\left(P_{k}\right)$ if and only if $v_{k}(x)$ is a positive solution of

$\left(P_{k, v}\right) \quad\left\{\begin{array}{l}-\Delta v-2 \nabla \ln w \cdot \nabla v=\left(\lambda g(x)-\mu g_{1}(x)\right) v \quad \text { on } B_{k}(0) ; \\ v=k w^{-1} \quad \text { on } \partial B_{k}(0) .\end{array}\right.$

Set $c(x)=\lambda g(x)-\mu g_{1}(x)$. Since $g(x) \leq g_{1}(x)$ on $R^{n}, g_{1}(x) \geq 0,0<\lambda \leq \mu$, we see that

$$
c(x)=\lambda g(x)-\mu g_{1}(x) \leq \lambda g_{1}(x)-\mu g_{1}(x) \leq \mu g_{1}(x)-\mu g_{1}(x)=0 .
$$

Thus we only need to show that the following problem has a positive solution $v_{k}(x)$ :

$$
\left(P_{k, v}\right) \quad\left\{\begin{array}{l}
\Delta v+2 \nabla \ln w \cdot \nabla v+c(x) v=0 \quad \text { on } \quad B_{k}(0) ; \\
v=k w^{-1} \quad \text { on } \quad \partial B_{k}(0) .
\end{array}\right.
$$

The existence of a solution follows immediately from Theorem 6.8 on p.100 in [8]. The positivity of the solution follows from Hopf's maximum principle (Theorem 3.5 on p. 35 in [8]) since $v_{k}>0$ on the boundary of $B_{k}(0)$. Therefore $\left(P_{k}\right)$ has a positive solution $u_{k}(x)$. We normalize $u_{k}(x)$ by setting $u_{k}(0)=1$. Then

$$
\left\{\begin{array}{l}
-\Delta u_{k}=\lambda g(x) u_{k} \quad \text { on } \quad B_{k}(0) \\
u_{k}(x)>0 \quad \text { on } \quad B_{k}(0) \\
u_{k}(0)=1
\end{array}\right.
$$

Now we apply the Harnack inequality for positive solutions of elliptic equations (Theorem 8.20 on p. 199 in [8]) to (2.2); we see that for any compact set $\Omega$ on $R^{n}$, there are constants $K$ and $M$ (where $M$ depends only on $\Omega, \lambda$, and $g(x) ; K$ depends only on $\Omega$ ), such that

$$
0<u_{k}(x) \leq M \quad \text { on } \quad \Omega \text { for } k \geq K .
$$

Then an application of the interior Schauder estimates (Theorem 6.2 on p. 90 in [8]) for solutions of elliptic equations yields

$$
\left|u_{k}\right|_{C^{2, \beta}(\Omega)} \leq M_{1} \quad \text { for } \quad k \geq K_{1}>K,
$$

where $\left|u_{k}\right|_{C^{2, \beta}(\Omega)}$ is the $C^{2, \beta}$ norm of $u_{k}, M_{1}, K_{1}$ and $\beta$ are constants (where $M_{1}$ depends only on $\Omega, \lambda$ and $g(x) ; \beta$ depends only on $\lambda$ and $g(x) ; K_{1}$ depends only on $\Omega$ ). Then it is clear that $u_{k}(x)$ has a subsequence which converges to a $C^{2}$ non-negative function $u(x)$ on $R^{n}$ on any compact set. Therefore

$$
-\Delta u(x)=\lambda g(x) u(x) \quad \text { on } \quad R^{n}, \quad u(0)=1, \quad u(x) \geq 0 .
$$

Now an application of a maximum principle concludes that $u(x)$ is positive everywhere on $R^{n}$. 
As we mentioned before, Theorem 2 is a result in [1]. For the convenience of our readers, we present a proof here using a slightly different approach. For a domain $\Omega$ on $R^{n}$, we denote $H_{0}^{1}(\Omega)$ the Banach space of all functions that have square integrable first order derivatives and are zero on the boundary.

Proof of Theorem 2. Let $B_{k}(0)$ be the ball centered at the origin with radius $k$, $k=1,2,3, \cdots$ By assumption, $g_{1}\left(x_{0}\right)>0$ for some $x_{0} \in R^{n}$. We may assume $x_{0} \in B_{1}(0)$. We consider a family of eigenvalue problems:

$$
\begin{cases}-\Delta u= & \lambda g_{1}(x) u \quad \text { on } \quad B_{k}(0) \\ u=0 & \text { on } \quad \partial B_{k}(0) .\end{cases}
$$

Let

$$
\lambda_{1}(k)=\inf \left\{\int_{B_{k}(0)}|\nabla u|^{2} d x \quad \mid u \in H_{0}^{1}\left(B_{k}(0)\right), \int_{B_{k}(0)} g_{1}(x) u^{2}=1\right\} .
$$

Since $g\left(x_{0}\right)>0$, we see that $\lambda_{1}(k)$ is well defined, non-increasing on $k$ and $\lambda_{1}(k) \geq 0$. Thus $\mu=\lim _{k \longrightarrow \infty} \lambda_{1}(k)$ exists. Furthermore it is easy to see that there is a nonnegative function $u_{k}(x) \in H_{0}^{1}\left(B_{k}(0)\right)$ that achieves the infimum. By the regularity theory for solutions of elliptic equations (Theorem 3.55 on p.86 in [2]) and the maximum principle, we see that $u_{k}(0)$ is actually a $C^{2}$ positive solution of $\left(D_{k}\right)$. We normalize $u_{k}(x)$ so that $u_{k}(0)=1$. Then

$$
\left\{\begin{array}{l}
-\Delta u_{k}=\lambda_{1}(k) g_{1}(x) u_{k} \quad \text { on } \quad B_{k}(0) ; \\
u_{k}(x)>0 \quad \text { on } \quad B_{k}(0) ; \\
u_{k}(0)=1 .
\end{array}\right.
$$

Now exactly as we did in the proof of Theorem 1, we see that $u_{k}(x)$ has a subsequence which converges to a $C^{2}$ non-negative function $u(x)$ on $R^{n}$ on any compact set. And $u(x)$ is a positive eigenfunction with the principal eigenvalue $\mu$.

To complete the proof, we only need to show that $\mu$ is bounded away from zero by the bound given in the statement of Theorem 2 .

By the Hölder inequality, we have

$$
\begin{gathered}
\int_{B_{k}(0)} g(x) u^{2} d x \leq \int_{B_{k}(0)} g^{+}(x) u^{2} d x \\
\leq\left(\int_{B_{k}(0)}\left|g^{+}(x)\right|^{\frac{n}{2}} d x\right)^{\frac{2}{n}}\left(\int_{B_{k}(0)}|u|^{\frac{2 n}{n-2}} d x\right)^{\frac{n-2}{n}} .
\end{gathered}
$$

Then if $\int_{B_{k}(0)} g(x) u^{2} d x=1$, we have

$$
\left(\int_{B_{k}(0)}\left|g^{+}(x)\right|^{\frac{n}{2}} d x\right)^{-\frac{2}{n}} \leq\left(\int_{B_{k}(0)}|u|^{\frac{2 n}{n-2}} d x\right)^{\frac{n-2}{n}} .
$$

On the other hand, by the Sobolev imbedding inequality (Theorem 2.14 on p. 39 in [2]), there is a constant $c_{1}(n)$, depending only on the dimension $n$, such that for all $u \in H_{0}^{1}\left(B_{k}(0)\right)$,

$$
\left(\int_{B_{k}(0)}|u|^{\frac{2 n}{n-2}} d x\right)^{\frac{n-2}{2 n}} \leq c_{1}(n)\left(\int_{B_{k}(0)}|\nabla u|^{2} d x\right)^{\frac{1}{2}}
$$


Thus

$$
\left(\int_{B_{k}(0)}\left|g^{+}(x)\right|^{\frac{n}{2}} d x\right)^{-\frac{2}{n}} \leq c_{1}^{2}(n)\left(\int_{B_{k}(0)}|\nabla u|^{2} d x\right) .
$$

This immediately yields

$$
\lambda_{1}(k) \geq c(n)\left(\int_{B_{k}(0)}\left|g^{+}(x)\right|^{\frac{n}{2}} d x\right)^{-\frac{2}{n}} \geq c(n)\left(\int_{R^{n}}\left|g^{+}(x)\right|^{\frac{n}{2}} d x\right)^{-\frac{2}{n}} .
$$

Therefore

$$
\mu \geq c(n)\left(\int_{R^{n}}\left|g^{+}(x)\right|^{\frac{n}{2}} d x\right)^{-\frac{2}{n}}
$$

Remark 2.1. The proofs of Theorems 1 and 2 can also be modified to cover more general cases. Let $\Omega$ be an unbounded domain in $R^{n}(n \geq 3)$ with $C^{2, \beta}$ boundary. We consider the principal eigenvalue problem for a second order linear elliptic operator in divergent form:

$$
\left\{\begin{array}{l}
-\frac{\partial}{\partial x_{i}}\left(a_{i j}(x) \frac{\partial u}{\partial x_{j}}\right)=\lambda g(x) u \quad \text { on } \quad \Omega \\
u=0 \quad \text { on } \partial \Omega \\
u(x)>0 \text { on } \Omega
\end{array}\right.
$$

Here the summation convention is used. We also assume that the coefficients are Hölder continuous and the equation is uniformly elliptic, that is, there are positive constants $m_{1}$ and $m_{2}$, such that

$$
m_{1}|\eta|^{2} \leq a_{i j}(x) \eta_{i} \eta_{j} \leq m_{2}|\eta|^{2} \quad \text { for } \quad \eta=\left(\eta_{1}, \eta_{2}, \cdots, \eta_{n}\right) \in R^{n}, \quad x \in R^{n} .
$$

Then a direct modification of the proofs of Theorems 1 and 2 yields

Theorem 9. If $n \geq 3, g^{+}\left(x_{0}\right)>0$ for some $x_{0} \in \Omega$, and

$$
\int_{\Omega}\left|g^{+}\right|^{\frac{n}{2}} d x<\infty
$$

then any number $\lambda$ is a principal eigenvalue of $\left(P_{1}\right)$ as long as

$$
0<\lambda \leq c\left(n, m_{1}, m_{2}\right)\left(\int_{\Omega}\left|g^{+}\right|^{\frac{n}{2}} d x\right)^{-\frac{2}{n}},
$$

where $c\left(n, m_{1}, m_{2}\right)$ is a constant depending only on $n, m_{1}, m_{2}$.

Sketch of the proof. In the proof of Theorem 1, we replace $B_{k}(0)$ in $\left(P_{k}\right)$ by $\Omega_{k}=$ $B_{k}(0) \cap \Omega$. Then we choose a function $h_{k}(x)$ on $\partial \Omega_{k}$ such that $h_{k}(x) \geq 0, h_{k}(x)=0$ on $\partial \Omega \cap \partial \Omega_{k}$, and $h_{k}(x)>0$ on part of $B_{k}(0) \cap \partial \Omega_{k}$. Also, let $u=h_{k}(x)$ on $\partial B_{k}(0)$ in $\left(P_{k}\right)$.

In the proof of Theorem 2, we replace $B_{k}(0)$ in $\left(D_{k}\right)$ by $\Omega_{k}=B_{k}(0) \cap \Omega$.

Those are all the changes needed to carry out the complete proof of Theorem 9. (Actually one might need to smooth out the edges of $\Omega_{k}$; we leave it to reader.)

Now we prove Theorem 5 . 
Proof of Theorem 5. We choose a radially symmetric smooth positive function $\eta(x)$ such that $\frac{c}{|x|^{2}} \geq \eta(x) \geq g_{1}(x)$ on $R^{n}$ and $\eta(x)=\frac{c}{|x|^{2}}$ for $|x|$ large. If we can show Theorem 5 for $\eta(x)$, we can apply Theorem 1 to $g_{1}(x)$ to get the conclusion.

It is easy to see that the Euler equation

$$
v^{\prime \prime}(r)+\frac{n-1}{r} v^{\prime}(r)+\frac{(n-2)^{2}}{4 r^{2}} v(r)=0
$$

has a solution $v(r)=r^{-\frac{n-2}{2}}$ for $0<r<\infty$. Thus $v(x)=v(|x|)$ satisfies

$$
-\Delta v=\frac{(n-2)^{2}}{4|x|^{2}} v \quad \text { on } \quad R^{n} \backslash\{0\} .
$$

Now for any number $0<\lambda \leq \frac{(n-2)^{2}}{4 c}$, we consider an initial value problem for the ordinary differential equation:

$$
\left\{\begin{array}{l}
\psi^{\prime \prime}(r)+\frac{n-1}{r} \psi^{\prime}(r)+\lambda \eta(r) \psi(r)=0, \\
\psi(0)=1, \psi^{\prime}(0)=0 .
\end{array}\right.
$$

Since $\eta(r)$ is smooth, it is clear that $(2.4)$ has a solution $\psi(r)$ defined on $(0, T)$ for some $T>0$. Also $\eta(r)>0$ implies that $\psi(r)$ is decreasing on $(0, T)$. We claim that $\psi(r)>0$ on $(0, T)$. Assume the claim for the moment. Then $\psi(r)$ can be extended beyond $T$, that is, $\psi(r)$ exists on $0<r<\infty$. Thus $\psi(r)>0$ is an eigenfunction corresponding to the principal eigenvalue $\lambda$.

We now prove that claim. Indeed if $\psi(r)<0$ for some $0<r<T$, since $\psi(0)=1$, we can find a $r_{0}$ such that $\psi\left(r_{0}\right)=0, \psi(r)>0$ for $0<r<r_{0}$. Then for $\psi(x)=\psi(|x|)$

$$
\left\{\begin{array}{lcc}
-\Delta \psi=\lambda \eta(|x|) \psi & \text { on } & 0<|x|<r_{0} \\
\psi(0)=1, \psi(x)=0 & \text { if } & |x|=r_{0} .
\end{array}\right.
$$

We then set $\psi(|x|)=\phi(|x|) v(|x|)$ for a new function $\phi(|x|)$. (2.3), (2.5) and a calculation shows that $\phi$ satisfies

$$
\left\{\begin{array}{l}
-\Delta \phi-2 \nabla \ln v \cdot \nabla \phi=\left(\lambda \eta(|x|)-\frac{(n-2)^{2}}{4|x|^{2}}\right) \phi(|x|), \\
\phi(0)=0, \phi(|x|)=0 \quad \text { if } \quad|x|=r_{0} \\
\phi(x)>0 \text { for } 0<|x|<r_{0} .
\end{array}\right.
$$

But $\lambda \leq \frac{(n-2)^{2}}{4 c}$ and the construction of $\eta(x)$ imply

$$
\lambda \eta(|x|) \leq \frac{\lambda c}{|x|^{2}} \leq \frac{(n-2)^{2}}{4|x|^{2}} \quad \text { for } \quad 0<|x|<r_{0} .
$$

Then we apply Hopf's maximum principle (Theorem 3.71 on p.96 in [2]) to (2.6) to conclude that $\phi(|x|)$ can not take a positive maximum on $0<|x|<r_{0}$ unless $\phi$ is a constant. This is a contradiction.

Remark 2.2. Since $\eta(r)=\frac{c}{r^{2}}$ for $r$ large, from (2.4) and the formula for general solutions of Euler equation, we see that if $0<\lambda<\frac{(n-2)^{2}}{4 c}$,

$$
\psi(r)=c_{1} r^{\alpha_{1}}+c_{2} r^{\alpha_{2}} \text { for } r \text { large, }
$$


where $c_{1}, c_{2}$ are constants and

$$
\alpha_{1}=\frac{1}{2}\left(-(n-2)+\sqrt{(n-2)^{2}-4 \lambda c}\right), \quad \alpha_{2}=\frac{1}{2}\left(-(n-2)-\sqrt{(n-2)^{2}-4 \lambda c}\right) .
$$

Therefore for different $\lambda$, the corresponding principal eigenfunction has different asymptotic behavior. For some particular principal eigenvalue, the asymptotic behavior of the corresponding eigenfunction is investigated in Theorem 7 .

Now we show Theorem 6 , which says that the assumptions in Theorem 5 are quite sharp.

Proof of Theorem 6 . We prove the conclusion by contradiction. Suppose that there is a $\lambda_{0}>0$ and a function $u(x)>0$, such that

$$
-\Delta u(x)=\lambda_{0} g(x) u(x) \quad \text { on } \quad R^{n}
$$

Fix a number $M>\frac{(n-2)^{2}}{4}$; then by the assumption of the theorem we can choose a number $M_{1}$ such that

$$
\lambda_{0} g(x) \geq \frac{M}{|x|^{2}} \quad \text { for } \quad|x| \geq M_{1} .
$$

Let $k$ be an integer greater than $M_{1}$. We consider a boundary value problem for an ordinary differential equation

$$
\left\{\begin{array}{l}
-v^{\prime \prime}(r)-\frac{n-1}{r} v^{\prime}(r)=\frac{\mu}{r^{2}} v(r) \quad \text { for } \quad M_{1}<r<k \\
v\left(M_{1}\right)=0, \quad v(k)=0 ; \\
v(r)>0 \text { for } \quad M_{1}<r<k .
\end{array}\right.
$$

Since the equation is an Euler equation, we see that only for

$$
\mu=\frac{(n-2)^{2}}{4}+\pi^{2} \ln ^{-2}\left(\frac{k}{M_{1}}\right),
$$

(2.8) has a solution of the form

$$
v_{k}(r)=C r^{-\frac{(n-2)}{2}} \sin \left(\sqrt{\mu-\frac{(n-2)^{2}}{4}} \ln \frac{r}{M_{1}}\right) .
$$

Thus $\mu \longrightarrow \frac{(n-2)^{2}}{4}$ as $k \longrightarrow \infty$. Now we fix a $k$ such that the corresponding $\mu<M$. Let $v_{k}(r)$ be the solution defined by $(2.9)$ with $C=1$. We set $v_{k}(x)=v_{k}(|x|)=$ $\phi_{k}(x) u(x)$ for a new function $\phi_{k}(x)$; a calculation shows that $\phi_{k}(x)$ satisfies

$$
\left\{\begin{array}{l}
-\Delta \phi_{k}(x)-2 \nabla \ln u(x) \cdot \nabla \phi_{k}(x)=\left(\frac{\mu}{|x|^{2}}-\lambda_{0} g(x)\right) \phi_{k}(x) \\
\phi_{k}(x)=0, \text { for } \quad|x|=M_{1} \\
\phi_{k}(x)=0, \text { for } \quad|x|=k \\
\phi_{k}(x)>0 \text { for } \quad M_{1}<|x|<k
\end{array}\right.
$$

But (2.7) and the choice of $\mu$ imply that

$$
\frac{\mu}{|x|^{2}}-\lambda_{0} g(x) \leq 0 \quad \text { for } \quad M_{1} \leq|x| \leq k
$$


Now an application of Hopf's maximum principle (Theorem 3.71 on p.96 in [2]) lets us conclude that $\phi_{k}(x)$ can not take an interior positive maximum unless $\phi_{k}(x)$ is a constant. This is a contradiction.

Now we prove Theorem 7 . The proof is carried out by pulling back the problem $(P)$ to an equivalent one on the unit sphere. Indeed, the proof of Theorem 7 includes that of Theorem 2. We have given a proof for Theorem 2 separately just for simplicity. Before we start the proof of Theorem 7, let us fix some notation. Let $s_{0}=(0,0, \cdots,-1)$ be the south pole of the unit sphere $S^{n}$ in $R^{n+1}, d s^{2}$ the standard metric on $S^{n}, d x^{2}$ the Euclidean metric on $R^{n}$. Use the stereographic projection $F: R^{n} \longrightarrow S^{n} \backslash\left\{s_{0}\right\}$,

$$
F(x)=\left(\frac{2 x}{1+|x|^{2}}, \frac{1-|x|^{2}}{1+|x|^{2}}\right), \quad x \in R^{n} .
$$

$S^{n} \backslash\left\{s_{0}\right\}$ is diffeomorphic to $R^{n}$, and under this coordinate chart

$$
d s^{2}=\frac{4}{\left(1+|x|^{2}\right)^{2}} d x^{2}, \quad x \in R^{n} .
$$

We denote $\psi(x)=4^{\frac{(n-2)}{4}}\left(1+|x|^{2}\right)^{-\frac{(n-2)}{2}}$; then $d s^{2}=\psi(x)^{\frac{4}{(n-2)}} d x^{2}$. The following lemma says that the eigenvalue problem $(P)$ can be translated into an equivalent eigenvalue problem on $S^{n}$. (For a function $v(x)$ on $R^{n}$, we still denote the corresponding function $v \circ F^{-1}$ by $v(x)$. The notation should be clear from the context.)

Lemma 2.1. $u(x)>0$ satisfies

$$
-\frac{4(n-1)}{n-2} \Delta u(x)=\lambda g(x) u(x) \quad \text { on } \quad R^{n}
$$

if and only if the function $w(x)=u(x) \psi^{-1}(x)$ satisfies

$$
-\frac{4(n-1)}{n-2} \Delta_{s} w+n(n-1) w=\lambda g \psi^{-\frac{4}{n-2}} w \quad \text { on } \quad S^{n} \backslash\left\{s_{0}\right\},
$$

where $\Delta_{s}$ is the Laplace-Beltrami operator on $S^{n}$ with respect to the metric $d s^{2}$.

Proof. $u(x)>0$ satisfies $(P)$ if and only if

$$
-\frac{4(n-1)}{n-2} \Delta u(x)=\lambda g(x) u^{-\frac{4}{n-2}}(x) u^{\frac{n+2}{n-2}}(x) \quad \text { on } \quad R^{n} .
$$

That is, the metric $u^{\frac{4}{n-2}}(x) d x^{2}$ has the scalar curvature function $\lambda g(x) u^{-\frac{4}{n-2}}(x)$. Therefore the metric $u^{\frac{4}{n-2}}(x) \psi^{-\frac{4}{n-2}} d s^{2}$ has the scalar function $\lambda g(x) u^{-\frac{4}{n-2}}(x)$. For $w(x)=u(x) \psi^{-1}$, this is equivalent to (see p. 126 in [2])

$$
-\frac{4(n-1)}{n-2} \Delta_{s} w+n(n-1) w=\lambda g u^{-\frac{4}{n-2}} w^{\frac{n+2}{n-2}} \quad \text { on } \quad S^{n} \backslash\left\{s_{0}\right\} .
$$

By the definition of $w(x)$, this is just $\left(P_{s}\right)$.

Now we are ready to prove Theorem 7 . The proof is more or less the same as that of Theorem 2. The difference here is that we are now working on the unit sphere $S^{n}$. Then we can use the fact that $S^{n}$ is a compact manifold. 
Proof of Theorem \%. Let $B_{\frac{1}{k}}\left(s_{0}\right)$ be the geodesic ball on $S^{n}$ centered at the south pole $s_{0}$ with radius $\frac{1}{k}, k=10,11, \cdots$. Denote $\Omega_{k}=S^{n} \backslash B_{\frac{1}{k}}\left(s_{0}\right)$. We may assume that $g\left(x_{0}\right)>0$ for some $x_{0} \in \Omega_{10}$. We consider

$$
\left(P_{s, k}\right) \quad\left\{\begin{array}{l}
-\frac{4(n-1)}{n-2} \Delta_{s} w+n(n-1) w=\lambda g \psi^{-\frac{4}{n-2}} w \text { on } \quad \Omega_{k} ; \\
w=0, \quad \text { on } \partial \Omega_{k} .
\end{array}\right.
$$

Let

$$
\begin{array}{r}
\lambda_{1}(k)=\inf \left\{\frac{1}{2} \int_{\Omega_{k}}\left(\frac{4(n-1)}{n-2}\left|\nabla_{s} w\right|^{2}+n(n-1) w^{2}\right) d V o l, \quad\right. \text { where } \\
\left.w \in H_{0}^{1}\left(\Omega_{k}\right), \int_{\Omega_{k}} g(x) \psi^{-\frac{4}{n-2}} w^{2} d V o l=1\right\} .
\end{array}
$$

where $d V o l$ is the volume form on $S^{n}$ induced from the metric $d s^{2}$ and $\nabla_{s}$ is the gradient with respect to the metric $d s^{2}$. Since $g\left(x_{0}\right)>0$ for some $x_{0}, \lambda_{1}(k)$ is well defined and there is a non-negative function $w_{k}(x) \in H_{0}^{1}\left(\Omega_{k}\right)$ that achieves the infimum. By the regularity theory for solutions of elliptic equations (Theorem 3.55 on p.86 in [2]) and the maximum principle, we see that $w_{k}(x)$ is actually a $C^{2}$ positive solution of $\left(P_{s, k}\right)$. Furthermore, from the definition of $\lambda_{1}(k)$, we see that $\lambda_{1}(k)$ is non-increasing on $k$ and $\lambda_{1}(k) \geq 0$; thus $\lambda_{0}=\lim _{k \rightarrow \infty} \lambda_{1}(k)$ exists. Now we have

$$
\lambda_{1}(k)=\frac{1}{2} \int_{\Omega_{k}}\left(\frac{4(n-1)}{n-2}\left|\nabla_{s} w_{k}\right|^{2}+n(n-1) w_{k}^{2}\right) d V o l
$$

and

$$
\int_{\Omega_{k}} g(x) \psi^{-\frac{4}{n-2}} w_{k}^{2} d V o l=1
$$

Thus a subsequence of $w_{k}(x)$ converges weakly in $H^{1}\left(S^{n}\right)$ to a non-negative function $w$ in $H^{1}\left(S^{n}\right)$. Then $w_{k}$ converges strongly to $w$ in $L^{q}\left(S^{n}\right)$ for any $q<\frac{2 n}{n-2}$. Since for some $p>\frac{n}{2}$,

$$
\int_{R^{n}}|g(x)|^{p}\left(1+|x|^{2}\right)^{2 p-n} d x<\infty
$$

we have

$$
\int_{S^{n}}|g|^{p} \psi^{-\frac{4 p}{n-2}} d V o l<\infty
$$

Then by the Hölder inequality,

$$
1=\int_{\Omega_{k}(0)} g(x) \psi^{-\frac{4}{n-2}} w_{k}^{2} d V o l \leq\left(\int_{S^{n}}|g|^{p} \psi^{-\frac{4 p}{n-2}} d V o l\right)^{\frac{1}{p}}\left(\int_{S^{n}} w_{k}^{2 q} d V o l\right)^{\frac{1}{q}}
$$

for some $q<\frac{n}{n-2}$. Thus

$$
\int_{S^{n}} g(x) \psi^{-\frac{4}{n-2}} w^{2} d V o l=\lim _{k \rightarrow \infty} \int_{\Omega_{k}} g(x) \psi^{-\frac{4}{n-2}} w_{k}^{2} d V o l=1 .
$$

On the other hand, it is easy to see that $w$ is a weak solution of

$$
\left\{\begin{array}{l}
-\frac{4(n-1)}{n-2} \Delta_{s} w+n(n-1) w=\lambda_{0} g \psi^{-\frac{4}{n-2}} w \text { on } S^{n} \\
w \geq 0 \text { on } S^{n} .
\end{array}\right.
$$


Since $g \psi^{-\frac{4}{n-2}} \in L^{p}\left(S^{n}\right)$ for some $p>\frac{n}{2}, w \in L^{\frac{2 n}{n-2}}\left(S^{n}\right)$, we have $g \psi^{-\frac{4}{n-2}} w \in$ $L^{q}\left(S^{n}\right)$ for some $q>\frac{n}{2}$ (this can be seen by Theorem 9.15 on p.241 in [8] and the bootstrap argument). Then the regularity theory for solutions of elliptic equations (Theorem 3.55 on p.86 in [2]) conclude that $w$ is smooth on $S^{n} \backslash\left\{s_{0}\right\}$ and continuous on $S^{n}$. An application of a maximum principle lets us conclude that $w(x)>0$ on $S^{n} \backslash\left\{s_{0}\right\}$. Therefore if $\lambda_{0}>0$, then $\lambda_{0}$ is a principal eigenvalue for $\left(P_{s}\right)$ and $w$ is a corresponding eigenfunction. We notice that for $(P), \lambda_{0}$ is a principal eigenvalue and $u(x)=w(x) \psi(x)$ is a corresponding principal eigenfunction. Moreover,

$$
u(x)|x|^{n-2}=w(x) \psi(x)|x|^{n-2} \longrightarrow w\left(s_{0}\right) \geq 0 \quad \text { as } \quad|x| \longrightarrow \infty .
$$

Now we still need to prove that $\lambda_{0}$ is bounded by the lower bound given in the statement of Theorem 7 . To do so, we only need to show that

$$
\lambda_{1}(k) \geq c(n)\left(\int_{R^{n}}\left|g^{+}\right|^{\frac{n}{2}} d x\right)^{-\frac{2}{n}} .
$$

For any $w \in H^{1}\left(S^{n}\right)$ (see Proposition 6.4 on p.126 and Theorem 2.14 on p.39 in $[2])$,

$$
\int_{S^{n}}\left(\frac{4(n-1)}{n-2}\left|\nabla_{s} w\right|^{2}+n(n-1) w^{2}\right) d V o l \geq c_{2}(n)\left(\int_{S^{n}} w^{\frac{2 n}{n-2}} d V o l\right)^{\frac{n-2}{n}}
$$

where $c_{2}(n)$ is a constant depending only on $n$. Also, by the Hölder inequality,

$$
\begin{gathered}
\int_{S^{n}} g(x) \psi^{-\frac{4}{n-2}} w^{2} d V o l \leq \int_{S^{n}} g^{+}(x) \psi^{-\frac{4}{n-2}} w^{2} d V o l \\
\leq\left(\int_{S^{n}}\left|g^{+}(x)\right|^{\frac{n}{2}} \psi^{-\frac{2 n}{n-2}} d V o l\right)^{\frac{2}{n}}\left(\int_{S^{n}}|w|^{\frac{2 n}{n-2}} d V o l\right)^{\frac{n-2}{n}},
\end{gathered}
$$

and finally we notice that

$$
\int_{S^{n}}\left|g^{+}(x)\right|^{\frac{n}{2}} \psi^{-\frac{2 n}{n-2}} d V o l=\int_{R^{n}}\left|g^{+}(x)\right|^{\frac{n}{2}} \psi^{-\frac{2 n}{n-2}} \psi^{\frac{2 n}{n-2}} d x=\int_{R^{n}}\left|g^{+}(x)\right|^{\frac{n}{2}} d x
$$

Then (2.10) is a direct consequence of (2.11), (2.12) and (2.13).

Remark 2.3. As we pointed out in Remark 2.2, Theorem 7 provides information on the asymptotic behavior only for a particular eigenfunction. The asymptotic behavior of other eigenfunctions (corresponding to other principal eigenvalues) is unknown.

If we in addition assume that $p>n$ in Theorem 7 , then the principal eigenfunction $u(x)$ obtained in the proof will satisfy

$$
u(x)|x|^{n-2} \longrightarrow c_{0} \geq 0 \quad \text { as } \quad|x| \longrightarrow \infty
$$

and

$$
|\nabla u||x|^{n-1} \longrightarrow c_{1} \quad \text { as } \quad|x| \longrightarrow \infty
$$

for some constants $c_{0}$ and $c_{1}$. 


\section{THE CASE THAT $n=2$}

Now we prove Theorem 8. As we did in the proof of Theorem 7 , we shall pull the equation in $(P)$ back onto the two dimensional unit sphere $S^{2}$. Let $s_{0}$ be the south pole of the unit sphere $S^{2}, d s^{2}$ the standard metric on $S^{2}, d x^{2}$ the Euclidean metric on $R^{2}$. By the stereographic projection $F: R^{2} \longrightarrow S^{2} \backslash\left\{s_{0}\right\}, S^{2} \backslash\left\{s_{0}\right\}$ is diffeomorphic to $R^{2}$, and under this coordinate chart

$$
d s^{2}=\frac{4}{\left(1+|x|^{2}\right)^{2}} d x^{2}, \quad x \in R^{2} .
$$

We denote $\psi(x)=4\left(1+|x|^{2}\right)^{-2}$; then $d s^{2}=\psi(x) d x^{2}$. The following lemma says that the eigenvalue problem can be translated into an equivalent eigenvalue problem on $S^{2}$. (Once again, we shall not distinguish the notation for functions on $R^{2}$ and $S^{2} \backslash\left\{s_{0}\right\}$ as we did in the proof of Theorem 7.)

Lemma 3.1. $u(x)$ satisfies

$$
-\Delta u(x)=\lambda g(x) u(x) \quad \text { on } \quad R^{2}
$$

if and only if the function $u(x)$ satisfies

$$
-\Delta_{s} u=\lambda g \psi^{-1} u \quad \text { on } \quad S^{2} \backslash\left\{s_{0}\right\},
$$

where $\Delta_{s}$ is the Laplace-Beltrami operator on $S^{2}$.

Proof. Under the coordinate chart (stereographic projection) $F: R^{2} \longrightarrow S^{2} \backslash\left\{s_{0}\right\}$,

$$
d s^{2}=\psi(x) d x^{2}, \quad x \in R^{2} .
$$

A direct calculation from the definition of the Laplace-Beltrami operator yields that

$$
\Delta_{s} u=\psi^{-1} \Delta u
$$

where $\psi(x)=4\left(1+|x|^{2}\right)^{-2}$.

Proof of Theorem 8. We write $g(x)=g^{+}(x)-g^{-}(x)$, where $g^{+}(x)=\max \{g(x), 0\}$ and $g^{-}(x)=\max \{-g(x), 0\}$. Now take a function $\phi(x) \in C_{0}^{\infty}\left(R^{2}\right)$ such that $\phi(x)=0$ for $|x|$ large, $0 \leq \phi(x) \leq 1$ on $R^{2}$, and

$$
\int_{R^{2}}\left(g^{+}(x)-\phi(x) g^{-}(x)\right) d x<0 .
$$

(3.1) is possible since we have assumed $\int_{R^{2}} g(x) d x<0$. We denote $g_{1}(x)=g^{+}(x)-$ $\phi(x) g^{-}(x)$; then $g_{1}^{+}(x)=g^{+}(x)$ and $g_{1}^{-}(x)=\phi(x) g^{-}(x)$, and (3.1) implies that

$$
\int_{S^{2}} g_{1} \psi^{-1} d V o l=\int_{R^{2}} g_{1} \psi^{-1} \psi d x=\int_{R^{2}} g_{1} d x<0 .
$$

Also the assumptions on $g$ imply

$$
\int_{R^{2}}\left|g_{1}(x)\right|^{p}\left(1+|x|^{2}\right)^{2(p-1)} d x<\infty,
$$

for some $p>2$. Then

$$
\begin{gathered}
\int_{S^{2}}\left|g_{1}(x) \psi^{-1}\right|^{p} d V o l=4^{1-p} \int_{R^{2}}\left|g_{1}(x)\right|^{p}\left(1+|x|^{2}\right)^{2 p}\left(1+|x|^{2}\right)^{-2} d x \\
=\int_{R^{2}}\left|g_{1}(x)\right|^{p}\left(1+|x|^{2}\right)^{2(p-1)}<\infty
\end{gathered}
$$


Then by Theorem 4.7 on p.104 in [2], (3.2) and (3.3), there is a function $z$ on $W^{2, p}\left(S^{2}\right) \cap C^{1, \beta}\left(S^{2}\right) \cap C^{2}\left(S^{2} \backslash\left\{s_{0}\right\}\right)$, such that

$$
\Delta_{s} z=g^{*}-g_{1} \psi^{-1} \quad \text { on } \quad S^{2} \backslash\left\{s_{0}\right\},
$$

where

$$
g^{*}=\frac{1}{4 \pi} \int_{S^{2}} g_{1} \psi^{-1} d V o l
$$

Thus we can choose a constant $\delta_{1}>0$ such that if $0<\delta<\delta_{1}$, then

$$
\begin{gathered}
\Delta_{s}(\delta z)+\left|\nabla_{s}(\delta z)\right|^{2}+\delta g(x) \psi^{-1}(x)=\delta \Delta_{s} z+\delta^{2}\left|\nabla_{s} z\right|^{2}+\delta g(x) \psi^{-1}(x) \\
=\delta g^{*}-\delta g_{1} \psi^{-1}+\delta^{2}\left|\nabla_{s} z\right|^{2}+\delta g(x) \psi^{-1}(x) \\
=\delta g^{*}-\delta\left(g_{1}^{+}(x)-g_{1}^{-}(x)\right) \psi^{-1}+\delta^{2}\left|\nabla_{s} z\right|^{2}+\delta\left(g^{+}(x)-g^{-}(x)\right) \psi^{-1}(x) \\
=\delta g^{*}-\delta\left(g^{+}(x)-g^{-}(x) \phi(x)\right) \psi^{-1}+\delta^{2}\left|\nabla_{s} z\right|^{2}+\delta\left(g^{+}(x)-g^{-}(x)\right) \psi^{-1}(x) \\
=\delta g^{*}+\delta^{2}\left|\nabla_{s} z\right|^{2}+\delta(\phi(x)-1) g^{-}(x) \psi^{-1}(x) \\
\leq \delta g^{*}+\delta^{2}\left|\nabla_{s} z\right|^{2} \leq 0 \quad \text { on } \quad S^{2} \backslash\left\{s_{0}\right\} ;
\end{gathered}
$$

here we have used the fact that $0 \leq \phi(x) \leq 1$. Then it is easy to check that $v=e^{\delta z}$ satisfies

$$
-\Delta_{s} v \geq \delta g(x) \psi^{-1} v \quad \text { on } \quad S^{2} \backslash\left\{s_{0}\right\} \quad \text { for } \quad 0<\delta<\delta_{1} .
$$

Now we start the same procedure as in the proof of Theorem 7 . That is, let $B_{\frac{1}{k}}\left(s_{0}\right)$ be the geodesic ball on $S^{2}$ centered at the south pole $s_{0}$ with radius $\frac{1}{k}, k=10,11, \cdots$. Denote $\Omega_{k}=S^{2} \backslash B_{\frac{1}{k}}\left(s_{0}\right)$. We may assume that $g\left(x_{0}\right)>0$ for some $x_{0} \in \Omega_{10}$. We consider

$$
\left\{\begin{array}{l}
-\Delta_{s} u=\lambda g \psi^{-1} u \quad \text { on } \quad \Omega_{k} \\
u=0, \quad \text { on } \partial \Omega_{k}
\end{array}\right.
$$

Let

$$
\lambda_{1}(k)=\inf \left\{\int_{\Omega_{k}}\left|\nabla_{s} u\right|^{2} d V o l \mid u \in H_{0}^{1}\left(\Omega_{k}\right), \quad \int_{\Omega_{k}} g(x) \psi^{-1} u^{2} d V o l=1\right\} .
$$

As we saw before, $\lambda_{1}(k)$ is well defined and achieved by a positive $C^{2}$ function $u_{k}(x) \in H_{0}^{1}\left(\Omega_{k}\right)$. Furthermore, $\lambda_{0}=\lim _{k \longrightarrow \infty} \lambda_{1}(k)$ exists. Now we normalize $u_{k}(x)$ such that $u_{k}\left(y_{0}\right)=1$ at the north pole $y_{0}$. As we did in the proof of Theorem 2 , we see that a subsequence of $u_{k}(x)$ converges in $C^{2}$ to a positive $C^{2}$ function $u(x)$ on any compact set on $S^{2} \backslash\left\{s_{0}\right\}$. Therefore if $\lambda_{0}>0, \lambda_{0}$ is a principle eigenvalue and $u(x)$ is a corresponding eigenfunction.

Now we show that $\lambda_{0}>0$. Let $\delta_{1}$ and $v(x)$ be defined in (3.4). We claim that $\lambda_{0} \geq \delta_{1}$. We only need to show that $\lambda_{1}(k) \geq \delta_{1}$ for $k$ large. Indeed if $\lambda_{1}(k)<\delta_{1}$ for some $k$, let $\delta=\lambda_{1}(k), v_{k}=e^{\lambda_{1}(k) z} ;(3.4)$ is

$$
-\Delta_{s} v_{k} \geq \lambda_{1}(k) g(x) \psi^{-1} v_{k} \quad \text { on } \quad S^{2} \backslash\left\{s_{0}\right\} .
$$


Set $u_{k}=v_{k} h_{k}$ for a new function $h_{k}$. Then the definition of $u_{k}(x)$ and (3.5) implies that $h_{k}$ satisfies

$$
\left\{\begin{array}{l}
-\Delta_{s} h_{k}-2\left\langle\nabla \ln v_{k}, \nabla h_{k}\right\rangle_{s} \leq 0 \text { on } \Omega_{k} \\
h_{k}=0 \text { on } \partial \Omega_{k} \\
h_{k}>0 \text { on } \Omega_{k} .
\end{array}\right.
$$

where $\left\langle\nabla \ln v_{k}, \nabla h_{k}\right\rangle_{s}$ is the inner product of the vectors $\nabla \ln v_{k}$ and $\nabla h_{k}$ under the metric $d s^{2}$. Now an application of Hopf's maximum principle (Theorem 3.71 on p.96 in [2]) to (3.6) concludes that $h_{k}$ can not take an interior positive maximum on $\Omega_{k}$ unless $h_{k}(x)$ is a constant. This is a contradiction.

Remark 3.1. The idea of constructing $v_{k}(x)$ in (3.5) is borrowed from [9].

\section{BIBLIOGRAPHIES}

1. Allegretto, W., Principal eigenvalues for indefinite weight elliptic problems in $R^{n}$, Proc. Amer. Math. Soc. 116 (1992), 701-706. MR 93a:35114

2. Aubin, T., Nonlinear analysis on manifolds, Springer-Verlag, New York (1982). MR 85j:58002

3. Brown, K.J., Cosner, C. \& Fleckinger, J., Principal eigenvalues for problems with indefinite weight function on $R^{n}$, Proc. Amer. Math. Soc. 109 (1990), 147-155. MR 90m:35140

4. Brown, K.J., Lin, S.S., \& Tertikas, A., Existence and nonexistence of steady-state solutions for a selection-migration model in population genetics, J. Math. Biol. 27 (1989), 91-104. MR 90i:92012

5. Brown, K.J. \& Tertikas, A., On the bifurcation of radially symmetric steady-state solutions arising in population genetics, SIAM J. Math. Anal. 22 (1991), 400-413. MR 92e:92018

6. Brown, K.J. \& Tertikas, A, The existence of principal eigenvalues for problems with indefinite weight function on $R^{k}$, Proc. Royal Soc. Edinburgh 123A (1993), 561-569. MR 94i:35136

7. Fleming, K.J., A selection-migration model in population genetics, J. Math. Biol. 2 (1975), 219-233. MR 53:7531

8. Gilbarg, D. \& Trudinger, N.S., Elliptic partial differential equations of second order, 2nd ed., Springer-Verlag, Berlin. (1983). MR 86c:35035

9. Jung, Y.T., On the elliptic equations $\frac{4(n-1)}{n-2} \Delta u+K(x) u^{\frac{n+2}{n-2}}=0$ and the conformal deformation of Riemannian metrics, Indiana Univ. Math. J. 43 (1994), 737-746. MR 96a:53052

Department of Mathematics and Statistics, Wichita State University, Wichita, KANSAS 67260

E-mail address: zhiren@cs.twsu.edu 